\title{
ACCELERATED STABILITY TESTING OF ANTI-AGING CREAM: FORMATION OF MYRISTIC ACID AND STEARIC ACID AS DEGRADATION PRODUCTS
}

\author{
ISKANDARSYAH, HARMITA*, AZZAHRA ALIYYA RAHMAN
}

Department of Pharmacy, Faculty of Pharmacy, Universitas Indonesia, Depok 16424, Indonesia. Email: igakadeharmita@gmail.com Received: 10 May 2018, Revised and Accepted: 01 October 2018

\begin{abstract}
Objective: This study aimed to determine the stability of anti-aging cream using the accelerated stability test and calculating the levels of myristic acid and stearic acid as degradation products.

Methods: Optimum conditions and validation methods for the mixture of myristic acid and stearic acid were determined to obtain a valid method for determining the levels of degradation products in anti-aging cream. Derivatization was performed with the Lepage esterification method, which uses methanol-toluene 4:1 (v/v) and acetyl chloride catalysts. The analysis was performed using gas chromatography (GC) Shimadzu GC-17A system with an HP-1 column and flame ionization detection.

Results: The column temperature was $120^{\circ} \mathrm{C}$, with an increase of $10^{\circ} \mathrm{C} / \mathrm{min}$ (up to $160^{\circ} \mathrm{C}$ ) followed by an increase of $3^{\circ} \mathrm{C} / \mathrm{min}$ (up to $220^{\circ} \mathrm{C}$; maintained for $5 \mathrm{~min}$ ) and an increase of $10^{\circ} \mathrm{C} / \mathrm{min}$ (up to $260^{\circ} \mathrm{C}$; maintained for $5 \mathrm{~min}$ ). The injector and detector temperatures were $260^{\circ} \mathrm{C}$ and $280^{\circ} \mathrm{C}$, respectively, with a flow rate of $1 \mathrm{~mL} / \mathrm{min}$. The retention times of myristic acid and stearic acid were $16.655 \mathrm{~min}$ and $28.169 \mathrm{~min}$, respectively, with tailing factor values of 0.734 and 0.943 , respectively. Validation results fulfilled our acceptance criteria, which obtained linearity for myristic acid at $y=9.6483+190.78 x$, with a correlation coefficient (r) value of 0.9997 , limit of detection (LOD) $=0.0013$ parts per million (ppm), and limit of quantification $(\mathrm{LOQ})=0.0042 \mathrm{ppm}$. The linearity for stearic acid was determined at $\mathrm{y}=17.163+106.22 \mathrm{x}$, with a correlation coefficient $(\mathrm{r}$ ) value of $0.9998, \mathrm{LOD}=0.0016 \mathrm{ppm}$, and $\mathrm{LOQ}=0.0053 \mathrm{ppm}$. Results of the anti-aging cream stability test indicated that the average remaining levels of isopropyl myristate from 0 to 3 months were $99.6283 \%, 99.1995 \%, 98.2571 \%$, and $97.1511 \%$, respectively. The average remaining levels of glyceryl monostearate were $99.6791 \%, 98.2881 \%, 96.2247 \%$, and $93.7195 \%$ from 0 to 3 months, respectively.
\end{abstract}

Conclusion: The expiration date of the anti-aging cream product was then calculated using zero-order kinetics and determined to be 10 months and 9 days.

Keywords: Myristic acid, Stearic acid, Isopropyl myristate, Glyceryl monostearate, Gas chromatography, Accelerated stability test, Validation, Optimization.

(C) 2018 The Authors. Published by Innovare Academic Sciences Pvt Ltd. This is an open access article under the CC BY license (http://creativecommons. org/licenses/by/4. 0/) DOI: http://dx.doi.org/10.22159/ijap.2018.v10s1.01

\section{INTRODUCTION}

Since ancient times, beauty has been a highly desirable trait, with skin being an outward reflection. Indeed, the skin is one part of the body where beauty can be more precisely defined. However, with age, the properties of skin can change. Indeed, extreme climate changes, high levels of pollution, smoking, hormonal changes, lifestyle, and ultraviolet (UV) exposure can all aggravate skin aging [1,2]. Characteristic signs of aging skin include reduced skin elasticity, wrinkles, and skin that appears to be thin [1].

At present, various techniques have been developed to prevent and treat skin aging, with one being the use of cosmetics [3]. Accordingly, cosmetics are widely used to overcome the problem of skin aging. Antiaging cosmetics function to rejuvenate the skin, help remove wrinkles, and protect the skin from oxidative damage caused by UV rays $[4,5]$. The anti-aging products currently available on the market are generally formulated as transparent creams and gels [6]. However, cream preparations are more popular, likely because they are easy to apply and easily removed from the skin [7].

Anti-aging cream products, as well as cosmetic creams in general, are composed of two main components, namely, the oil phase and the water phase. Cream preparations generally contain $>20 \%$ water content and $<50 \%$ of the oil phase [8]. Some ingredients often used as oils or as the lipophilic phase in creams include ester compounds of fatty acids, including sorbitan monooleate, polyoxyl 40 stearate, glyceryl dilaurate, glyceryl oleate, glyceryl monostearate (GMS), and isopropyl myristate [9].
GMS is one of the ester fatty acid compounds used in lotions and creams as an emulsifier, stabilizer, thickener, and emollient and is the most widely sold emulsifier commercially. In addition to GMS, isopropyl myristate is an important ingredient used in cosmetics, functioning as an emollient that can restore and retain the natural softness of the skin [10]. However, when the water content is high, as in the preparation of creams under acidic conditions, the ester bonds in both GMS and isopropyl myristate become broken, which leads to their break down into various degradation products. Accordingly, isopropyl myristate breaks down into isopropanol isopropyl myristate and myristic acid, while GMS decomposes into stearic acid and glycerol $[11,12]$. Based on this decomposition, the stability of anti-aging cream preparations can be evaluated.

The stability test is composed of a series of tests aimed at obtaining information on the stability of pharmaceutical products, to determine their shelf life and expiration date under certain packaging and storage conditions [13]. The long-term stability test is often performed to produce more significant degradation data for preparation [14]. However, this stability test takes a long time to determine the expiration date, which precludes timely cosmetic notification processes for production. Therefore, the preferred stability test is the accelerated method, which requires shorter test times, where the decomposition may cause the consistency of the cream to change $[13,14]$. Therefore, in this study, we performed the accelerated stability test at $40^{\circ} \mathrm{C} \pm 2^{\circ} \mathrm{C}$ and a relative humidity of $70 \pm 5 \%$ to accelerate the expiration date of 
the product. Data acquisition was based on the remaining isopropyl myristate and GMS in the product at $0,1,2$, and 3 months.

In previous studies, stability tests were conducted on cosmetic preparations using the accelerated method for 90 days, a temperature of $40^{\circ} \mathrm{C}$, and a relative humidity of $75 \%$. In addition, tests were performed at room temperature. After the test, the physical properties of the preparation, such as organoleptic, $\mathrm{pH}$, viscosity, and the levels of active substances were observed [2]. While there has been an increasing use of anti-aging creams, little data exist on the stability of the chemicals in these creams; thus, it is necessary to study the components of cream preparations.

In this study, accelerated stability testing was conducted on the preparation of anti-aging creams containing isopropyl myristate and GMS as their base components. The stability of the anti-aging cream was judged by determining the residual isopropyl myristate levels, based on the formation of myristic acid, and the residual GMS levels, based on the formation of stearic acid. Considering the fatty components of the cream, gas chromatography (GC) methods were chosen as the best technique. Indeed, GC is the method most often used for the analysis of fatty acids and is very sensitive for their identification and quantification [15]. However, fatty acids detected using GC processes first require derivatization to lower their boiling point, and the response to the detector used [16]. In this study, derivatization was performed using the Lepage esterification method, followed by analysis using GC.

\section{METHODS}

\section{Samples}

The anti-aging cream used for accelerated stability testing at $0,1,2$, and 3 months was composed of a formulation of glycolic acid (10\%), Lanette 0 (5\%), isopropyl myristate (5.5\%), GMS (4.5\%), emulgade SE-PF (3.5\%), propylene glycol (5\%), glycerin (5\%), methylparaben $(0.01 \%)$, propylparaben $(0.002 \%)$, and water $(\operatorname{ad} 100 \%)$.

\section{Chemical material}

A myristic acid standard (Natural Oleochemicals Sdn Bhd), stearic acid standard (Edenor), isopropyl myristate standard (Oleon), GMS standard (Evonik), acetyl chloride (Merck), methanol p.a (Merck), toluene p.a (Merck), potassium carbonate (Merck), nitrogen (UHP dan HP), and hydrogen (UHP) were all of reagent grade or higher.

\section{Chromatographic condition}

Shimadzu GC-17A systems are equipped with a flame ionization detector, a capillary column with a length of $30 \mathrm{~m}$ and an inside diameter of $0.25 \mathrm{~mm}$, film thickness of $0.25 \mu \mathrm{m}$ with a stationary phase HP-1, and carrier gases of nitrogen and hydrogen. The solution class GC data processor had an initial column temperature of $1200^{\circ} \mathrm{C}$, which was increased $10^{\circ} \mathrm{C} / \mathrm{min}$ (up to $160^{\circ} \mathrm{C}$ ), then raised $3^{\circ} \mathrm{C} / \mathrm{min}$ (up to $220^{\circ} \mathrm{C}$; maintained for $5 \mathrm{~min}$ ). Then, the temperature was raised $10^{\circ} \mathrm{C} / \mathrm{min}$ (up to $260^{\circ} \mathrm{C}$; maintained for $5 \mathrm{~min}$ ). The injector and detector temperatures were set at $260^{\circ} \mathrm{C}$ and $280^{\circ} \mathrm{C}$, respectively. The carrier gas flow rate was $1.0 \mathrm{~mL} / \mathrm{min}$.

Preparation of potassium carbonate solution (6\%)

Approximately $6 \mathrm{~g}$ of $\mathrm{K} 2 \mathrm{CO} 3$ was carefully weighed and placed into a $100 \mathrm{~mL}$ volumetric flask, followed by dilution with distilled water to mark the boundaries of the flask.

\section{Preparation of the myristic acid stock solution}

Approximately $25 \mathrm{~g}$ of myristic acid standard was carefully weighed and placed into a $25 \mathrm{~mL}$ volumetric flask followed by dilution with methanol-toluene $4: 1(\mathrm{v} / \mathrm{v})$ to mark the boundaries of the flask.

\section{Preparation of the stearic acid stock solution}

Approximately $25 \mathrm{~g}$ of the stearic acid standard was carefully weighed and placed into a $25 \mathrm{~mL}$ volumetric flask followed by dilution with methanol:toluene $4: 1(\mathrm{v} / \mathrm{v})$ to mark the boundaries of the flask.

\section{Determination of optimum conditions}

To determine the optimum conditions for testing, as much as $1.0 \mathrm{~mL}$ of each test solution of 4 parts per million ( $\mathrm{ppm}$ ) was pipetted and mixed. The solution was then placed in a test tube stoppered and shaken with a vortex. Acetyl chloride $(1 \mu \mathrm{L})$ was then added slowly while stirring. The tube was sealed while shaking occasionally. Furthermore, $5.0 \mathrm{~mL}$ of $6 \%$ potassium carbonate solution was slowly added. The tube was then tightly closed and centrifuged at $3000 \mathrm{rpm}$ for $5 \mathrm{~min}$. The top layer (toluene phase), containing the methyl ester mixture of myristic acid and stearic acid, was injected $(1 \mu \mathrm{L})$ into the GC system. Optimum conditions for analysis were assessed by retention time, the number of theoretical plates $(\mathrm{N})$, height equivalent to a theoretical plate, tailing factor, and the best separation resolution in Table 1.

\section{Analysis of the effect of heating time on placebo}

The placebo, weighing as much as $200 \mathrm{mg}$, was then fed into a $100 \mathrm{~mL}$ measuring flask and dissolved in $4: 1(\mathrm{v} / \mathrm{v})$ methanol: toluene, up to the boundary marker. Then, $200 \mu \mathrm{L}$ of acetyl chloride was added slowly while stirring. The tube was then placed into a water bath at $100^{\circ} \mathrm{C}$ for $10 \mathrm{~min}, 5 \mathrm{~min}$, and without heating. The tube was then sealed while shaking occasionally. $5 \mathrm{~mL}$ of $6 \%$ potassium carbonate solution was then slowly added. The tube was then tightly closed and centrifuged at $3000 \mathrm{rpm}$ for $5 \mathrm{~min}$. The top layer (toluene phase), containing the methyl ester mixture of myristic acid and stearic acid, was injected $(1 \mu \mathrm{L})$ into the GC system. Based on the results of our analysis, the peak area and comparative peak areas of myristic acid and stearic acid were recorded with each variation in conditions.

\section{Analysis of the addition of acetyl chloride to placebo}

The placebo, weighing as much as $200 \mathrm{mg}$, was then fed into a $100 \mathrm{~mL}$ measuring flask and dissolved in 4:1 (v/v) methanol: toluene, up to the boundary marker. Acetyl chloride was then added slowly while stirring, with the variation of addition volume being $10 \mu \mathrm{L}, 5 \mu \mathrm{L}$, and $1 \mu \mathrm{L}$. The tube was sealed while shaking occasionally. Furthermore, $5.0 \mathrm{~mL}$ of $6 \%$ potassium carbonate solution was slowly added. The tube was then tightly closed and centrifuged at $3000 \mathrm{rpm}$ for $5 \mathrm{~min}$. The top layer (toluene phase), containing the methyl ester mixture of myristic acid and stearic acid, was injected $(1 \mu \mathrm{L})$ into the GC system. Based on our results of the analysis, the peak areas of myristic acid and stearic acid were observed for each variation.

\section{System conformity test}

Sample preparation was performed in accordance with procedures for determining the optimum conditions for analysis. The injection of each solution was performed up to 6 times. The results of the analysis were recorded and the coefficient of variation was counted $(\% \mathrm{KV})$. The acceptance criteria of $\% \mathrm{KV}$ were $2 \%$ or less.

\section{Validation of the GC analytical method}

\section{Linearity test of myristic acid and stearic acid}

Test solutions of myristic acid and stearic acid (10 ppm) were prepared at $100 \mu \mathrm{L}$ and $500 \mu \mathrm{L}$. Aliquots (1.0, 2.0, 4.0, and $8.0 \mathrm{~mL}$ ) were then inserted into a $10 \mathrm{~mL}$ measuring flask. A sufficient volume of methanol:toluene solution $4: 1(\mathrm{v} / \mathrm{v})$ was used to obtain standard solutions of concentration: $0.1 ; 0.5 ; 1 ; 2 ; 4$; and 8 ppm.

\section{Detection limit test (LOD) and quantitation limit (LOQ)}

The LOD and LOQ were calculated statistically by linear regression calibration curves. The calculation results were equal to the value of

Table 1: Variation of initial column temperature and flow rate for determining the optimum conditions for analyzing myristic acid and stearic acid content

\begin{tabular}{ll}
\hline Initial column temperature $\left({ }^{\circ} \mathbf{C}\right)$ & Flow rate $(\mathbf{m L} / \mathbf{m i n u t e})$ \\
\hline 100 & 1.0 \\
120 & 1.2 \\
140 & 1.5 \\
\hline
\end{tabular}


Table 2: Optimization of analysis condition for mixture of myristic and stearic acid

Initial column temperature $\left({ }^{\circ} \mathrm{C}\right)$

\begin{tabular}{|c|c|c|c|c|c|c|c|c|c|}
\hline & \multicolumn{3}{|l|}{100} & \multicolumn{3}{|l|}{120} & \multicolumn{3}{|l|}{140} \\
\hline & \multicolumn{9}{|c|}{ Flow rate $(\mathrm{mL} / \mathrm{min})$} \\
\hline & 1.0 & 1.2 & 1.5 & 1.0 & 1.2 & 1.5 & 1.0 & 1.2 & 1.5 \\
\hline Retention time (min) & 19.057 & 18.001 & 16.842 & 16.655 & 15.438 & 14.218 & 13.629 & 12.477 & 11.166 \\
\hline Peak area $(\mu \mathrm{V} / \mathrm{s})$ & 689 & 557 & 804 & 749 & 658 & 1001 & 1302 & 1237 & 1587 \\
\hline Theoretical plate $(\mathrm{N})$ (plates) & 480866 & 424006 & 391146 & 391327 & 332864 & 232531 & 202990 & 185770 & 115155 \\
\hline $\operatorname{HETP}(\mathrm{cm})$ & 0.0062 & 0.0071 & 0.0077 & 0.0077 & 0.0090 & 0.0129 & 0.0147 & 0.0161 & 0.0260 \\
\hline Resolution & 163.61 & 163.36 & 159,61 & 145.00 & 145.17 & 115.35 & 55.51 & 51.082 & 44.616 \\
\hline (Tf) & 0.73 & 0.76 & 0.94 & 0.81 & 0.84 & 0.81 & 0.80 & 0.71 & 0.77 \\
\hline
\end{tabular}

$\mathrm{b}$ in the straight-line equation $\mathrm{y}=\mathrm{a}+\mathrm{bx}$, whereas the standard deviation of the blank was equal to the residual standard deviation (Sy/x) [17].

\section{Selectivity test}

The selectivity test was carried out by preparing a standard solution of myristic acid and stearic acid, and a derivatization solution with no standard added. Each solution was injected into the GC at $1 \mu \mathrm{L}$ under the selected analysis conditions. It was then observed whether or not other peaks appeared at the time of the retention of the methyl esters of myristic acid and stearic acid in the sample solution.

\section{Precision accuracy test}

The placebo cream, weighing as much as $200 \mathrm{mg}$, was added to each standard solution, then dissolved in a $100 \mathrm{~mL}$ volumetric flask using methanol:toluene $4: 1(\mathrm{v} / \mathrm{v})$. The volume of standard solution added was calculated to obtained concentrations of $80 \%(3.2 \mu \mathrm{g} / \mathrm{mL}$ ), $100 \%$ $(4 \mu \mathrm{g} / \mathrm{mL})$, and $120 \%(4.8 \mu \mathrm{g} / \mathrm{mL})$.

\section{RESULTS AND DISCUSSION}

\section{Analysis conditions optimization}

Based on our experiments, the resulting optimum analysis conditions for analyzing the mixture of myristic acid and stearic acid were: An initial temperature of $120^{\circ} \mathrm{C}$ for the column and a mobile phase flow rate of $1.0 \mathrm{~mL} / \mathrm{min}$ (Table 2). The resulting chromatogram had a fairly large peak area and relatively shorter retention time but displayed the best resolution when compared with other variations. The peak area obtained under these conditions was $749 \mu \mathrm{V} / \mathrm{s}$. The retention time obtained from the chromatogram of myristic acid was approximately $16.655 \mathrm{~min}$

For stearic acid, the peak area obtained under these conditions was $451 \mu \mathrm{V} / \mathrm{s}$. The retention time obtained from the chromatogram for myristic acid was $28.169 \mathrm{~min}$. The retention times for myristic acid and stearic acid are known and confirmed by the analysis of each substance under the selected analysis conditions, that is, the initial temperature of the column was $1200^{\circ} \mathrm{C}$ and the carrier gas flow rate was $1.0 \mathrm{~mL} / \mathrm{min}$. The resultant chromatogram was obtained and myristic acid appeared at a retention time of $16.251 \mathrm{~min}$, while stearic acid appeared at a retention time of $28.986 \mathrm{~min}$.

\section{System conformity test}

The system suitability test should be performed first before analyzing the optimum conditions selected. A number of variations made to the equipment, as well as the analytical techniques used, were evaluated. Thus, it is necessary to test the suitability of the system to ensure the effectiveness of the operational systems and to deliver results in accordance with the purpose of the analysis. The system suitability test was performed 6 times in a row and yielded coefficients of variation for the mixture of myristic acid and stearic acid of $0.9999 \%$ and $1.0433 \%$, respectively. Based on these experiments, our results matched the requirement that the coefficient of variation (KV) does not exceed $2 \%$.

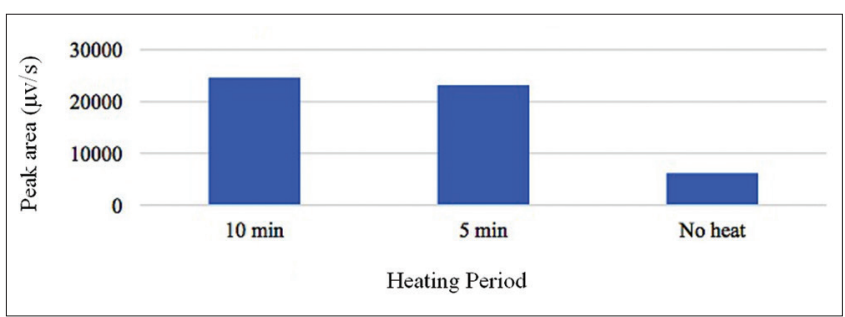

Fig. 1: Comparison of the peak area of myristic acid in the analysis of the effect of heating on the placebo

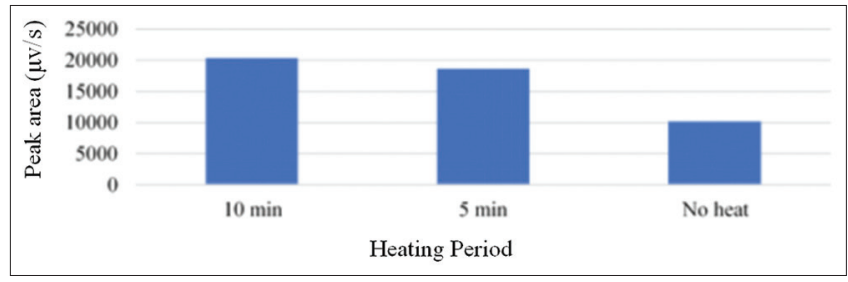

Fig. 2: Comparison of the peak area of stearic acid in the analysis of the effect of heating on the placebo

\section{Analysis of the effect of heating on placebo}

In this experiment, we analyzed the effect of heating on the placebobased cream containing isopropyl myristate and GMS. This analysis was performed by varying the Lepage esterification procedure used, that is, by heating at $1000^{\circ} \mathrm{C}$ for $10 \mathrm{~min}, 5 \mathrm{~min}$, and without heating. In this experiment, acetyl chloride as a catalyst was still used with an additional volume of $200 \mu \mathrm{L}$. The test results showed that the peak area of analysis using the same warming was slightly larger than those without the use of heating (Figs. 1 and 2). This likely occurred because in the presence of heating the reaction is catalyzed and may decompose into isopropyl myristate and GMS $[18,19]$. Thus, the chosen method of esterification without heating to reduce the effect of the reaction of isopropyl myristate and GMS was considered optimal.

Analysis of the addition of acetyl chloride to the placebo in conditions without heating

After analyzing the effect of heating on the placebo, we analyzed the effect of the amount of acetyl chloride added to the placebo. This analysis was conducted to determine whether the addition of acetyl chloride can cause isopropyl myristic and GMS to break down, which should not decompose during the preparation process. Variations in the volume used included $10 \mu \mathrm{L}, 5 \mu \mathrm{L}$, and $1 \mu \mathrm{l}$. Our test results showed that the peak area decreases with the increase in volume added (Figs. 3 and 4). Thus, the optional addition volume of $1 \mu \mathrm{L}$, due to the smaller amount of acetyl chloride added, may minimize the possibility of reacting with the remaining isopropyl myristate and GMS. In this case, the acid reacted only with myristic acid and stearic acid as a result of the decomposition. This may occur because acetyl 
Table 3: Chemical stability data of isopropyl myristate

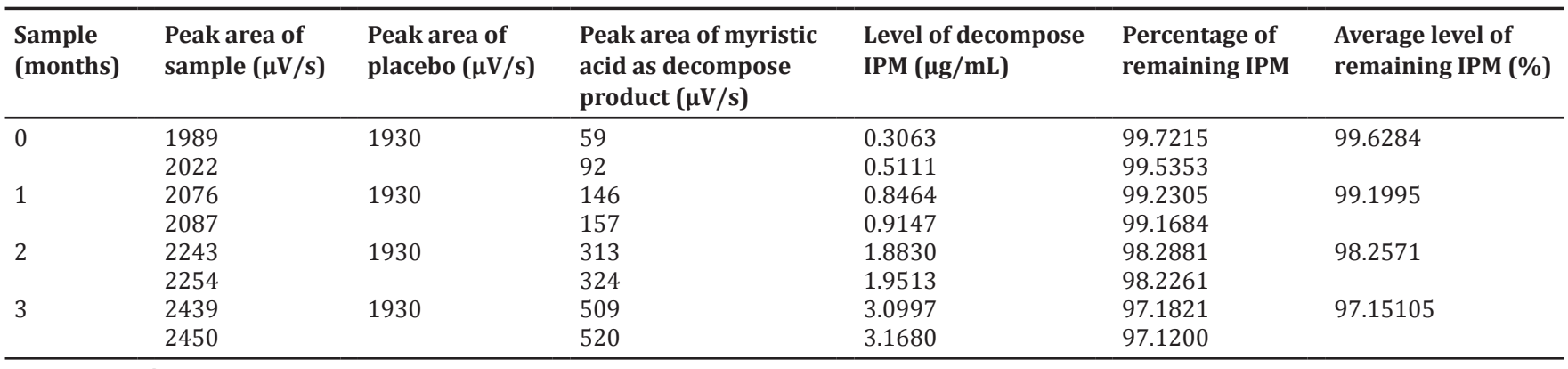

IPM: Integrated pest management

Table 4: Chemical stability data of GMS

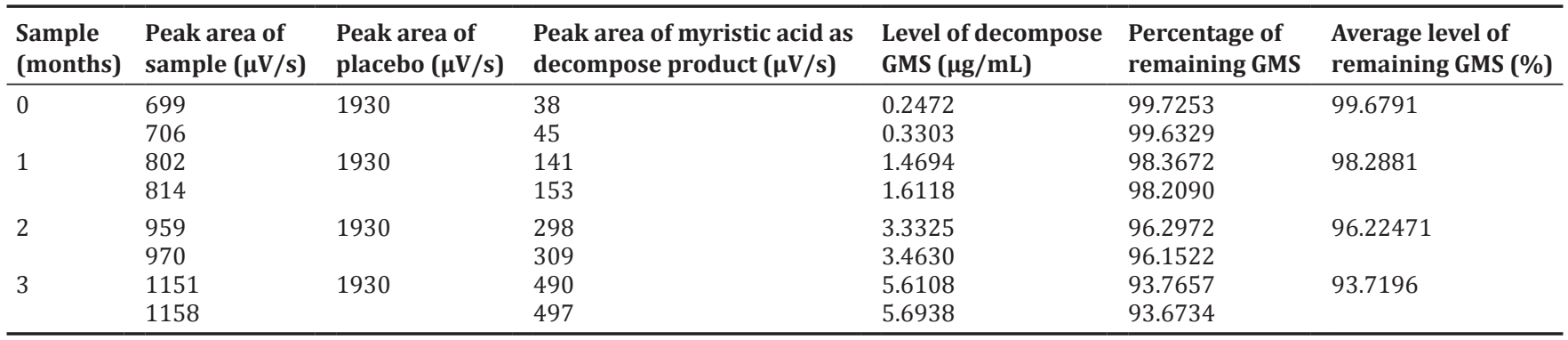

GMS: Glyceryl monostearate

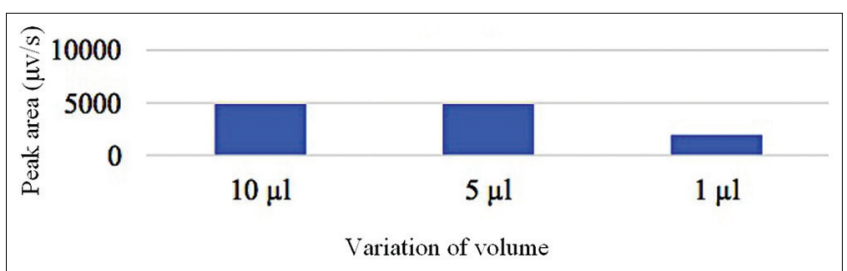

Fig. 3: Comparison of the peak area of myristic acid with additional volumes of acetyl chloride in the placebo

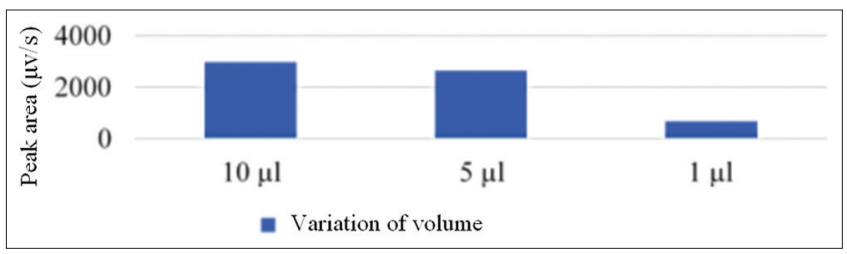

Fig. 4: Comparison of the peak area of stearic acid with additional volumes of acetyl chloride in the placebo

chloride is a strong acid that can catalyze the reaction, including both hydrolysis and esterification reactions [20].

\section{Validation of analysis methods}

Selectivity test

The selectivity test was performed by injecting the esterified product from the blank solution containing no fatty acids. The purpose of this test was to look at the possibility of a disturbance around the retention time of the fatty acid methyl esters. The results of these tests showed that the method was selective because there was no interference in the retention times of myristic acid and stearic acid.

\section{Linearity test}

The linearity test was performed by making a calibration curve by using six concentrations of the mixture of myristic acid and stearic acid, which were: $0.1,0.5,1.0,2.0,4.0$, and $8.0 \mathrm{ppm}$. The regression equations for myristic acid and stearic acid were $y=191.17 x+7.4638([r]=0.9997)$ and $y=107.06 x+12.482([r]=0.9996)$, respectively. Based on these results, the linearity test was declared valid because it met the linearity criteria of having a correlation coefficient of close to 1 or $>0.9990$.

\section{Accuracy and precision test}

Based on the results of our analysis, we calculated the percentage of recovery (UPK) by comparing the results of the analysis with the actual results. The criteria of accuracy were expressed as met if the percentage recovery (\% UPK) obtained was $98-102 \%$. The precision was determined as a relative standard deviation or coefficient of variation $(\mathrm{KV})$. The criteria of the precision were considered met if the method used produced a coefficient of variation (KV) of $2 \%$ or less.

Based on the results of our analysis, we obtained a value of recovery (UPK) for myristic acid and stearic acid of approximately 99.74$101.98 \%$ and $98.19-101.51 \%$, respectively. The value of the coefficient of variation (KV) produced for myristic acid and stearic acid was 0.49$0.89 \%$ and $1.14-1.79 \%$, respectively. Based on these results, it can be stated that the method used to analyze the mixture of myristic acid and stearic acid met the criteria of precision (accuracy) and precision.

Existence test of free myristic acid and stearic acid on isopropyl myristic and GMS

In this study, we tested the existence of free myristic acid and stearic acid in isopropyl myristate and GMS. Based on the chromatogram of the analytical results obtained, we did not observe any free myristic acid or stearic acid in the standard isopropyl myristate and GMS. This is consistent with the information contained in the data sheet for each substance, which does not indicate the presence of free myristic acid or stearic acid in isopropyl myristic and GMS.

\section{Data stability analysis of anti-aging cream samples}

In this study, we analyzed the stability of samples of anti-aging creams after $0,1,2$, and 3 months. Before the analysis, each sample was dissolved first using a 4:1 (v/v) methanol:toluene solution, then reacted with acetyl chloride $(1 \mu \mathrm{L})$ and no heating. After that, $5.0 \mathrm{~mL}$ 
of $6 \%$ solution of potassium carbonate was added and the solution was centrifuged at $3000 \mathrm{rpm}$ for $5 \mathrm{~min}$. The toluene layer formed was then injected into the GC system at $1 \mu \mathrm{L}$.

In this study, the Lepage esterification procedure used was modified for the amount of added acetyl chloride reagent, that is, from $200 \mu \mathrm{L}$ to $1 \mu \mathrm{L}$ and without the use of heating. This was due to the excessive use of acetyl chloride and heating time for $60 \mathrm{~min}$, which can cause GMS to decompose entirely into stearic acid, as well as isopropyl myristate to decompose entirely into myristic acid. Reactions that occur as a result of the hydrolysis of the ester compound can lead to the ester compound decomposing into a carboxylic acid in the presence of a catalyst, in the form of heat or acid. If such reactions occur in a cream base that does not decompose, it can hinder the process of decomposition analysis and the stability preparation test. In samples from the monthly stability tests, there were fatty acids, namely myristic acid and stearic acid, present into the $3^{\text {rd }}$ month. The levels of myristic acid and stearic acid contained in each sample as a result of decomposition were calculated using a linear regression equation with concentrations corresponding to the range of the obtained content.

Based on our analysis results the levels of isopropyl myristate and GMS decreased from 0 to 3 months. Thus, the average level of isopropyl myristate remaining in the sample was equal to $99.6283 \%, 99.1995 \%$, $98.2571 \%$, and $97.1511 \%$ from months 0 to 3, respectively (Table 3). The average GMS content remaining was 99.6791\%, 98.2881\%, $96,2247 \%$, and $93.7195 \%$ from months 0 to 3, respectively (Table 4). Increased levels of isopropyl myristate and GMS were found to degrade into myristic acid and stearic acid, which can occur due to the hydrolysis reaction of the ester compound. This reaction occurs due to the water content contained in the cream, and the glycolic acid aids as a catalyst to accelerate the reaction. Thus, the longer the carboxylic acid ester compound is exposed to the water content and the acid catalyst, the more the ester compound decomposes and forms the carboxylic acid, that is, free fatty acids.

\section{CONCLUSION}

The results of the anti-aging cream stability test indicated that the average remaining levels of isopropyl myristate from 0 to 3 months were $99.6283 \%, 99.1995 \%, 98.2571 \%$, and $97.1511 \%$, respectively. The remaining levels of GMS were 99.6791\%, 98.2881\%, 96.2247\%, and 93.7195 from 0 to 3 months, respectively. The expiration date of the anti-aging cream product was calculated using zero-order kinetics and determined to be 10 months and 9 days. Increased levels of isopropyl myristate and GMS degraded into myristic acid and stearic acid occur due to the hydrolysis reaction of the ester compound.

\section{CONFLICTS OF INTEREST}

All authors have none to declare.

\section{REFERENCES}

1. Li X. Anti-aging cosmetics and its efficacy assessment methods. IOP Conf Ser: Mater Sci Eng 2015;87(1):1-5.

2. Srivastava DRS, Shah KP. Formulation and evaluation of novel herbal anti-ageing formulation (gel-cream). World $\mathrm{J}$ Pharm Res 2015;4(8):2426-44.

3. Weiss RA, Weiss MA. Evaluation of a novel anti-aging topical formulation containing cycloastragenol, growth factors, peptides, and antioxidants. J Drugs Dermatol 2014;13(9):1135-9.

4. Hahn HJ, Jung HJ, Schrammek-Drusios MC, Lee SN, Kim JH, Kwon SB, et al. Instrumental evaluation of anti-aging effects of cosmetic formulations containing palmitoyl peptides, Silybum marianum seed oil, vitamin $\mathrm{E}$ and other functional ingredients on aged human skin. Exp Ther Med 2016;12(2):1171-76.

5. Gianeti MD, Maia Campos PMBGM. Efficacy evaluation of a multifunctional cosmetic formulation: The benefits of a combination of active antioxidant substances. Molecules 2014;19(11):18268-82.

6. Bhasha SA, Khalid SA, Duraivel S, Bhowmik D, Kumar KPS. Recent trends in usage of polymers in the formulation of dermatological gels. Indian J Res Pharm Biotechnol 2013;1(2):161-68.

7. Allen LV, Ansel HC. Ansel's pharmaceutical dosage forms and drug delivery systems $10^{\text {th }}$ ed. Wolter Kluwer health: United States of America; 2014: pp.102-299.

8. Fahr A. Voigt's pharmaceutical technology $1^{\text {st }}$ ed. Hoboken: John Wiley \& Sons Ltd; 2018: pp. 279-340.

9. Niazi SK. Handbook of pharmaceutical manufacturing formulations $2^{\text {nd }}$ ed. Boca Raton: CRC Press; 2009.

10. Johnson DH. The use of fatty acid derivatives in cosmetics and toiletries. J Am Oil Chem Soc 1978;55(4):438-43.

11. Diamante C, Fiume MZ, Bergfeld WF, Belsito DV, Hill RA, Klaassen CD, et al. Final safety assessment of thiodipropionic acid and its dialkyl esters as used in cosmetics. Int J Toxicol 2010;29(4): 137S-150S.

12. Bradley, Coulier. An investigation into the reaction and breakdown products from starting substances used to produce food contact plastic. London; 2007.

13. Manurung J. Drug quality assurance: Compendium guidelines and related materials, $1^{\text {st }}$ ed. Jakarta: Penerbit Buku Kedokteran EGC; 2006.

14. Bajaj S, Sakhuja N, Singla D, Bajaj Principal S. Stability testing of pharmaceutical products. J Appl Pharm Sci 2012;2(3):129-38.

15. Fisk HL, West AL, Childs CE, Burdge GC, Calder PC. The use of gas chromatography to analyze compositional changes of fatty acids in rat liver tissue during pregnancy. J Vis Exp 2014;85:1-10.

16. Zhang H, Wang Z, Liu O. Development and validation of a GC-FID method for quantitative analysis of oleic acid and related fatty acids. J Pharm Anal 2015;5(4):223-30.

17. Harmita, MJ.Physico-chemical analysis. Jakarta: Penerbit Buku Kedokteran EGC; 2015. [In Indonesian]

18. Carey FA, Sundberg RJ. Advanced organic chemistry part A: Structure and mechanisms. US: Springer; 2007.

19. Stoker HS. Organic and biological chemistry $7^{\text {th }}$ ed. Boston: Cengage Learning; 2015.

20. Johnson WA. Invitation to organic chemistry. London: Jones \& Bartlett Learning; 1999. 